\title{
Fast transport of two ions in an anharmonic trap
}

\author{
M. Palmero, ${ }^{1}$ E. Torrontegui, ${ }^{1}$ D. Guéry-Odelin, ${ }^{2}$ and J. G. Muga ${ }^{1,3}$ \\ ${ }^{1}$ Departamento de Química Física, Universidad del País Vasco-Euskal Herriko Unibertsitatea, Apdo. 644, Bilbao, Spain \\ ${ }^{2}$ Laboratoire de Collisions Agrégats Réactivité, CNRS UMR 5589, IRSAMC, Université de Toulouse (UPS), 118 Route de Narbonne, \\ 31062 Toulouse CEDEX 4, France \\ ${ }^{3}$ Department of Physics, Shanghai University, 200444 Shanghai, People's Republic of China \\ (Received 22 September 2013; revised manuscript received 8 October 2013; published 21 November 2013)
}

\begin{abstract}
We design fast trajectories of a trap to transport two ions using a shortcut-to-adiabaticity technique based on invariants. The effects of anharmonicity are analyzed first perturbatively, with an approximate, single relativemotion mode, description. Then, we use classical calculations and full quantum calculations. This allows us to identify discrete transport times that minimize excitation in the presence of anharmonicity. An even better strategy to suppress the effects of anharmonicity in a continuous range of transport times is to modify the trajectory using an effective trap frequency shifted with respect to the actual frequency by the coupling between relative and center-of-mass motions.
\end{abstract}

DOI: 10.1103/PhysRevA.88.053423

PACS number(s): 37.10.Ty, 03.67.Lx

\section{INTRODUCTION}

Quantum information processing based on trapped ions may be applied to a large number of qubits (and become scalable) by moving the ions between fixed zones where logic operations are performed [1-4]. The transport should be fast, but excitations should also be avoided at the destination site. Different approaches have been proposed to implement faster-than-adiabatic transport of cold atoms [5-11]. Diabatic transport of cold neutral atoms was demonstrated by GuéryOdelin and co-workers [7] and, recently, fast transport of single or two trapped ions was also realized by two groups [12-14]. One of the proposed approaches makes use of invariants to design trap trajectories without final excitation $[6,10,11,15]$. It is very flexible and provides by construction, under specific conditions, a motionally unexcited final transported state. It also allows for further trajectory optimization taking into account different experimental constraints, and robustness versus noise [16]. The invariant-based inverse engineering method has been applied so far to model the fast transport of a single particle $[10,11]$ and Bose-Einstein condensates [6]. In this paper, we extend the theoretical analysis in [10] to two Coulomb-interacting particles within a single trap, focusing on the effects of a mild anharmonicity which is present in any experimental setting [5,17]. In Sec. II, we study the transport of two ions first in a harmonic trap and then in an anharmonic trap with an added time-dependent linear potential to compensate the inertial force. The applicability of this compensating method may be limited so other options are explored. In particular, we consider in Sec. III the effect of anharmonicity when the trap trajectories are designed for an unperturbed (harmonic) trap. This is done using an approximate one-dimensional (1D) theory combined with perturbation theory. In Sec. IV, we study numerically the full two-dimensional (2D) problem. The article ends with a discussion in Sec. V and an appendix on the extension of some of the results to the transport of $N$ ions.

\section{TWO-ION TRANSPORT}

\section{A. Harmonic trap}

Let us examine first the transport of two single-charge ions of mass $m$ in an effectively one-dimensional harmonic trap that moves from 0 to $d$ in a time $t_{f}$. Let $\mathrm{q}_{1}$ and $\mathrm{q}_{2}$ be the coordinates of the two ions with momenta $\mathrm{p}_{1}$ and $\mathrm{p}_{2}$ and $Q_{0}(t)$ the trajectory of the trap minimum. We use first the laboratory frame and distinguish operators from $c$ number with a hat. The Hamiltonian includes a kinetic term, a harmonic potential, and an interaction potential due to the Coulomb force

$$
\begin{aligned}
\widehat{H}= & \frac{\widehat{\mathrm{p}}_{1}^{2}}{2 m}+\frac{\widehat{\mathrm{p}}_{2}^{2}}{2 m}+\frac{1}{2} m \omega^{2}\left[\left(\widehat{\mathrm{q}}_{1}-Q_{0}\right)^{2}+\left(\widehat{\mathrm{q}}_{2}-Q_{0}\right)^{2}\right] \\
& +\frac{C_{c}}{\widehat{\mathrm{q}}_{1}-\widehat{\mathrm{q}}_{2}} .
\end{aligned}
$$

$\omega /(2 \pi)$ is the trap frequency and $C_{c}=\frac{e^{2}}{4 \pi \epsilon_{0}}$, where $e$ is the electron charge and $\epsilon_{0}$ the vacuum permittivity. Here and in the following, we omit frequently the time argument of the trap position, i.e., $Q_{0}=Q_{0}(t)$. We set $\mathrm{q}_{1}>\mathrm{q}_{2}$ because of the strong Coulomb repulsion. The wave functions of the ions never superpose, so we may effectively treat the particles as distinguishable and the symmetrization of the wave function is not necessary as it will not provide any new physical effect. This assumption is largely accepted when interpreting current experiments.

Let us now introduce coordinates and momenta, as well as corresponding operators, for center-of-mass (CM) and relative motion,

$$
\begin{aligned}
\widehat{Q}=\frac{1}{2}\left(\widehat{\mathrm{q}}_{1}+\widehat{\mathrm{q}}_{2}\right) ; & \widehat{P}=\widehat{\mathrm{p}}_{1}+\widehat{\mathrm{p}}_{2}, \\
\widehat{r}=\frac{1}{2}\left(\widehat{\mathrm{q}}_{1}-\widehat{\mathrm{q}}_{2}\right) ; & \widehat{p}=\widehat{\mathrm{p}}_{1}-\widehat{\mathrm{p}}_{2} .
\end{aligned}
$$

This gives equal effective masses for relative and $\mathrm{CM}$ motions. The generalization for $N$ ions (see the Appendix) also holds this property. Substituting the new coordinates in Eq. (1), the 
Hamiltonian takes the form

$$
\begin{aligned}
\widehat{H}(\widehat{Q}, \widehat{P}, \widehat{r}, \widehat{p})= & \frac{\widehat{P}^{2}}{2 M}+\frac{1}{2} M \omega^{2}\left(\widehat{Q}-Q_{0}\right)^{2} \\
& +\frac{\widehat{p}^{2}}{2 M}+\frac{1}{2} M \omega^{2} \widehat{r}^{2}+\frac{C_{c}}{2 \widehat{r}},
\end{aligned}
$$

where $M=2 m$ is the total mass. The Hamiltonian is the sum of two terms $\widehat{H}=\widehat{H}_{\mathrm{cm}}+\widehat{H}_{r}$, where each term depends only on one of the pairs' coordinate momentum. We may thus "separate variables" and find time-dependent solutions of the Schrödinger equation.

The relative part of the Hamiltonian $\widehat{H}_{r}$ does not depend on $Q_{0}(t)$ so the relative motion is not affected by the transport and will remain unexcited. Thus, we only need to design a trajectory for which the CM is unexcited at final time. This may be achieved adiabatically or via shortcuts to adiabaticity. The CM Hamiltonian $\widehat{H}_{\mathrm{cm}}$ has the form of a particle of mass $M$ in a harmonic trap, so any of the shortcut-to-adiabaticy techniques known (using fast forward, optimal control, invariants, or their combination [5,8-10]) may be applied to find a suitable $Q_{0}(t)$.

To inverse engineer the trap trajectory making use of invariants, the invariant is designed first, consistent with a predetermined structure of the Hamiltonian [10]. The invariant is parametrized by the classical trajectory $Q_{c}(t)$ that satisfies the classical equation of motion $\ddot{Q}_{c}+\omega^{2}\left(Q_{c}-Q_{0}\right)=0$ and boundary conditions $Q_{c}(0)=\dot{Q}_{c}(0)=\ddot{Q}_{c}(0)=Q_{0}(0)=0$; $Q_{c}\left(t_{f}\right)=Q_{0}\left(t_{f}\right)=d ; \dot{Q}_{c}\left(t_{f}\right)=\ddot{Q}_{c}\left(t_{f}\right)=0$. They imply the initial and final commutativity between the invariant and the Hamiltonian, and the stability of the solution when the Hamiltonian remains constant beyond the boundary times. A simple polynomial interpolation gives [10]

$$
\begin{aligned}
& Q_{c}=d\left(10 s^{3}-15 s^{4}+6 s^{5}\right), \\
& Q_{0}=\frac{d}{\omega^{2} t_{f}^{2}}\left(60 s-180 s^{2}+120 s^{3}\right)+d\left(10 s^{3}-15 s^{4}+6 s^{5}\right),
\end{aligned}
$$

where $s=t / t_{f}$. Each initial eigenstate of $\widehat{H}_{\mathrm{cm}}(0)$ would evolve exactly according to the "transport mode"

$$
\Psi_{n}(Q, t)=e^{-\frac{i}{\hbar}\left[E_{n} t+\int_{0}^{t} \frac{M \dot{Q}_{c}^{2}}{2} d t^{\prime}\right]} e^{i M \dot{Q}_{c} Q / \hbar} \Phi_{n}\left(Q-Q_{c}\right)
$$

where $\Phi_{n}(x)$ are the eigenfunctions of the harmonic oscillator and $E_{n}$ the corresponding energies. At $t_{f}$ the modes become again eigenstates of the Hamiltonian $\widehat{H}\left(t_{f}\right)$, but at intermediate times they are in general a superposition of several eigenstates of $\widehat{H}(t)$. Note that, apart from transport between stationary states, it is also possible to design launching protocols, in which the system begins at rest and ends up with a given centerof-mass velocity, and, similarly, stopping protocols [10].

The separability between CM and relative motions is still valid for two ions of different masses if they oscillate with the same trapping frequency, but it breaks down if the frequency depends on position $Q_{0}$, if the two ions experience different trapping frequencies, or in presence of anharmonicity. We shall concentrate on this latter case, as it occurs in all traps and affects neutral atoms as well.

\section{B. Anharmonic trap}

We now consider an additional quartic potential in the Hamiltonian

$$
\begin{aligned}
\widehat{H}= & \frac{\widehat{\mathrm{p}}_{1}^{2}}{2 m}+\frac{\widehat{\mathrm{p}}_{2}^{2}}{2 m}+\frac{C_{c}}{\widehat{\mathrm{q}}_{1}-\widehat{\mathrm{q}}_{2}}+\frac{1}{2} m \omega^{2}\left[\left(\widehat{\mathrm{q}}_{1}-Q_{0}\right)^{2}\right. \\
& \left.\left.+\left(\widehat{\mathrm{q}}_{2}-Q_{0}\right)^{2}+\beta\left(\widehat{\mathrm{q}}_{1}-Q_{0}\right)^{4}+\beta \widehat{\mathrm{q}}_{2}-Q_{0}\right)^{4}\right]
\end{aligned}
$$

where $\beta$ is a perturbative constant with dimensions $[L]^{-2}$ that sets the "strength" of the anharmonicity. Nonrigid transport with a time-dependent trap frequency or time-dependent anharmonicities due to noise or control limitations is clearly of interest, but we shall only address here rigid transport as a first simpler step before considering more ambitious goals.

In terms of $\mathrm{CM}$ and relative coordinates we have

$$
\begin{aligned}
\widehat{H}= & \frac{\widehat{P}^{2}}{2 M}+\frac{1}{2} M \omega^{2}\left[\left(\widehat{Q}-Q_{0}\right)^{2}+\beta\left(\widehat{Q}-Q_{0}\right)^{4}\right] \\
& +\frac{\widehat{p}^{2}}{2 M}+\frac{1}{2} M \omega^{2}\left(\widehat{r}^{2}+\beta \widehat{r}^{4}\right)+\frac{C_{c}}{2 \widehat{r}} \\
& +3 M \omega^{2} \beta\left(\widehat{Q}-Q_{0}\right)^{2} \widehat{r}^{2} \\
= & \widehat{H}_{\mathrm{cm}}+\widehat{H}_{r}+\widehat{H}_{c} .
\end{aligned}
$$

The first two lines of Eq. (7) may be identified as (perturbed) $\mathrm{CM}$ and relative Hamiltonians $\widehat{H}_{\mathrm{cm}}$ and $\widehat{H}_{r}$. Unlike the harmonic trap, there is now a coupling term $\widehat{H}_{c}$ (third line) that depends both on $\widehat{Q}$ and $\widehat{r}$ so the variables can not be separated. No nontrivial invariants are known for this Hamiltonian $[18,19]$, so in principle we can not inverse-engineer the trap trajectory exactly using invariants. One approximate option is to design it for the unperturbed harmonic oscillator. An exact alternative is to apply a linear potential to compensate the inertial force as in $[10,20,21]$.

\section{Compensating force approach}

In this section we introduce an additional time-dependent linear term in the Hamiltonian to compensate for the effect of the trap motion in the trap frame and avoid final excitations. This generalizes for two ions the results in [10]. The extension of the compensating force approach to $N$ ions was discussed by Masuda in [20] using the fast-forward approach, and may also be carried out following the Appendix.

Let us first define a unitary transformation $[10,22]$ that shifts the momentum and position of the center-of-mass coordinate

$$
\widehat{\mathcal{U}}=e^{i \widehat{P} Q_{0}(t) / \hbar} e^{-i M \dot{Q}_{0}(t) \widehat{Q} / \hbar} .
$$

This amounts to change the reference system from a laboratory frame to the rest frame of the trap. ${ }^{1}$

We first rewrite the Hamiltonian in the laboratory frame as

$$
\widehat{H}\left(\widehat{Q}-Q_{0}, \widehat{r}, \widehat{P}, \widehat{p}\right)=\frac{\widehat{P}^{2}}{2 M}+\frac{\widehat{p}^{2}}{2 M}+\widehat{U}\left(\widehat{Q}-Q_{0}, \widehat{r}\right),
$$

where $\widehat{U}\left(\widehat{Q}-Q_{0}, \widehat{r}\right)$ can be any arbitrary potential. The equation for the transformed (trap frame) wave function

\footnotetext{
${ }^{1}$ Since $\widehat{P}$ and $\widehat{Q}$ do not commute, alternative orderings are possible but they only change the Hamiltonian by purely time-dependent terms without physical effect.
} 
$|\Phi\rangle=\widehat{\mathcal{U}}|\Psi\rangle$ takes the form ${ }^{2}$

$$
\begin{aligned}
i \hbar \partial_{t}|\Phi\rangle & =\widehat{H}_{\text {trap }}|\Phi\rangle \\
& =\left[\widehat{H}(\widehat{Q}, \widehat{r}, \widehat{P}, \widehat{p})+M\left(\widehat{Q}+Q_{0}\right) \ddot{Q}_{0}+\frac{1}{2} M \dot{Q}_{0}^{2}\right]|\Phi\rangle,
\end{aligned}
$$

where $\widehat{H}_{\text {trap }}=\widehat{\mathcal{U}} \widehat{H} \widehat{\mathcal{U}}^{\dagger}+i \hbar\left(\partial_{t} \widehat{\mathcal{U}}\right) \widehat{\mathcal{U}}^{\dagger}$. To compensate the inertial term $M\left(\widehat{Q}+Q_{0}\right) \ddot{Q}_{0}$ in the trap frame we may apply, in the laboratory frame, the term

$$
-M \widehat{Q} \ddot{Q}_{0}
$$

or, equivalently, a force $m \ddot{Q}_{0}$ on each particle. To make the term $\frac{1}{2} M \dot{Q}_{0}^{2}$ disappear, we may perform a further transformation $\left|\Phi^{\prime}(t)\right\rangle=\widehat{\mathcal{U}}^{\prime}(t)|\Phi(t)\rangle$, with $\widehat{\mathcal{U}}^{\prime}(t)=e^{\frac{i}{\hbar} \int_{0}^{t} \frac{1}{2} M \dot{Q}_{0}^{2} d t^{\prime}}$. This is independent of all operators, so that $\widehat{\mathcal{U}}^{\prime} \widehat{H}_{\text {trap }} \widehat{\mathcal{U}}^{\prime \dagger}$ does not modify the Hamiltonian, but $i \hbar\left(\partial_{t} \widehat{\mathcal{U}}^{\prime}\right) \widehat{\mathcal{U}}^{\prime \dagger}=-M \dot{Q}_{0}^{2} / 2$. We finally get

$$
i \hbar \partial_{t}\left|\Phi^{\prime}\right\rangle=\left[\frac{\widehat{P}^{2}}{2 M}+\frac{\widehat{p}^{2}}{2 M}+\widehat{U}(\widehat{Q}, \widehat{r})\right]\left|\Phi^{\prime}\right\rangle
$$

The resulting potential does not depend anymore on time, and any stationary state in the rest frame of the trap will remain so during transport. This holds for arbitrary potentials, even if $\widehat{Q}$ and $\widehat{r}$ are coupled, as in Eq. (7).

A lower bound for the maximum acceleration of the compensating force is $2 d / t_{f}^{2}$ [10]. Since the forces that can be applied are typically limited by experimental constraints, the compensation is not always easy to implement in practice, if at all. For this reason, we study in the following alternative strategies. First, we shall design the trap motion for an unperturbed harmonic potential and analyze the effect of anharmonicity.

\section{1D APPROXIMATION}

In this section, we discuss a simple approximation that provides valuable hints, even in analytical form, on the transport behavior of two ions in presence of anharmonicities. The idea is to freeze the relative motion coordinate at $r=r_{e}$, the minimum of the potential part that depends on $r$ only. Equivalently, we may consider a single-mode approximation in which relative-motion excitations are neglected. Neglecting constant terms, the resulting Hamiltonian has the same form as the one for the frozen relative coordinate, substituting $r_{e}$ and $r_{e}^{2}$ by the average values $\langle\widehat{r}\rangle$ and $\left\langle\widehat{r}^{2}\right\rangle$ in the ground relative-motion mode. With our parameters, the average and minimum values of $r$ are equal up to the third significant number, so the difference is negligible and we use for simplicity the frozen values.

With this assumption, and adding a constant term without physical effect, the Hamiltonian (7) becomes

$\widehat{H}=\frac{\widehat{P}^{2}}{2 M}+\frac{1}{2} M \omega^{2}\left[\left(6 \beta r_{e}^{2}+1\right)\left(\widehat{Q}-Q_{0}\right)^{2}+\beta\left(\widehat{Q}-Q_{0}\right)^{4}\right]$,

\footnotetext{
${ }^{2}$ Use $e^{-i M \dot{Q}_{0} \widehat{Q} / \hbar} \widehat{P} e^{i M \dot{Q}_{0} \widehat{Q} / \hbar}=\widehat{P}+M \dot{Q}_{0}$ and $e^{i Q_{0} \widehat{P} / \hbar} \widehat{Q} e^{-i Q_{0} \widehat{P} / \hbar}=$ $\widehat{Q}+Q_{0}$.
}

which we may also write as $\widehat{H}=\widehat{H}_{0}+\beta \widehat{H}_{1}$, where $\widehat{H}_{1}$ is a perturbation of the harmonic Hamiltonian $\widehat{H}_{0}$ :

$$
\widehat{H}_{1}=\frac{1}{2} M \omega^{2}\left[6 r_{e}^{2}\left(\widehat{Q}-Q_{0}\right)^{2}+\left(\widehat{Q}-Q_{0}\right)^{4}\right] .
$$

Let the initial state be $\left|\Psi_{n}(0)\right\rangle$. Using time-dependent perturbation theory, the final wave vector $\left|\Psi\left(t_{f}\right)\right\rangle$ is given by $[10,16,23]$

$$
\begin{aligned}
&\left|\Psi\left(t_{f}\right)\right\rangle \widehat{U}_{0}\left(t_{f}, 0\right)\left|\Psi_{n}(0)\right\rangle \\
& \quad-\frac{i \beta}{\hbar} \int_{0}^{t_{f}} d t \widehat{U}_{0}\left(t_{f}, t\right) \widehat{H}_{1}(t)\left|\Psi_{n}(t)\right\rangle \\
& \quad-\frac{\beta^{2}}{\hbar^{2}} \int_{0}^{t_{f}} d t \int_{0}^{t} d t^{\prime} \widehat{U}_{0}\left(t_{f}, t\right) \widehat{H}_{1}(t) \widehat{U}_{0}\left(t, t^{\prime}\right) \widehat{H}_{1}\left(t^{\prime}\right)\left|\Psi_{n}\left(t^{\prime}\right)\right\rangle \\
& \quad+O\left(\beta^{3}\right),
\end{aligned}
$$

where $\widehat{U}_{0}$ is the unperturbed propagator for $\widehat{H}_{0}$. In terms of the complete set of transport modes [see Eq. (5)], it takes the form

$$
\widehat{U}_{0}\left(t, t^{\prime}\right)=\sum_{j}\left|\Psi_{j}(t)\right\rangle\left\langle\Psi_{j}\left(t^{\prime}\right)\right| .
$$

To calculate the fidelity $F:=\left|\left\langle\Psi_{n}\left(t_{f}\right) \mid \Psi\left(t_{f}\right)\right\rangle\right|$ up to second order it is useful to separate the sum into $j=n$ and $j \neq n$ terms in the second-order contribution of Eq. (15). When computing $\left|\left\langle\Psi_{n}\left(t_{f}\right) \mid \Psi\left(t_{f}\right)\right\rangle\right|^{2}$, the square of first-order terms is canceled by the second-order term with $j=n$. Thus, the fidelity, up to second order, may finally be written as

$$
F=\left(1-\sum_{j \neq n}\left|f_{j, n}^{(1)}\right|^{2}\right)^{1 / 2},
$$

where $f_{j, n}^{(1)}=\frac{-i \beta}{\hbar} \int_{0}^{t_{f}} d t\left\langle\Psi_{j}(t)\left|\widehat{H}_{1}(t)\right| \Psi_{n}(t)\right\rangle .^{3}$ Due to the orthogonality properties of the Hermite polynomials, transitions induced by the quadratic perturbation will only be nonzero for one- and two-level jumps. Instead, the quartic part of the perturbation will lead to jumps from one to four levels. The $f_{j, n}^{(1)}$ transition amplitudes can be explicitly calculated so that the second-order fidelity is known analytically, although the form is too lengthy to be displayed here. Simplified expressions will be provided later. We compare the fidelity in second order with the exact, numerical one (using the split-operator method) in Fig. 1, starting both with the ground state of the harmonic trap $\Phi_{0}(0)$ and the exact ground state of the anharmonic trap. The results are hardly distinguishable. In the numerical examples we use the parameters in [12] except for a lower trap frequency to enhance anharmonic effects. The trap trajectory $Q_{0}(t)$ is chosen as in Eq. (4), using invariant-based engineering for the unperturbed system with a polynomial ansatz for $Q_{c}$. The fidelity oscillates, reaching the maximum value of one at discrete values of $t_{f}$. The occurrence of maxima is a generic feature that does not depend on the specific value of $\beta$ chosen. In the following, we work out a theory to explain and predict them.

\footnotetext{
${ }^{3}$ Similarly, the final average phonon number is $\langle j\rangle=n+$ $\sum_{j \neq n}(j-n)\left|f_{j, n}^{(1)}\right|^{2}$.
} 


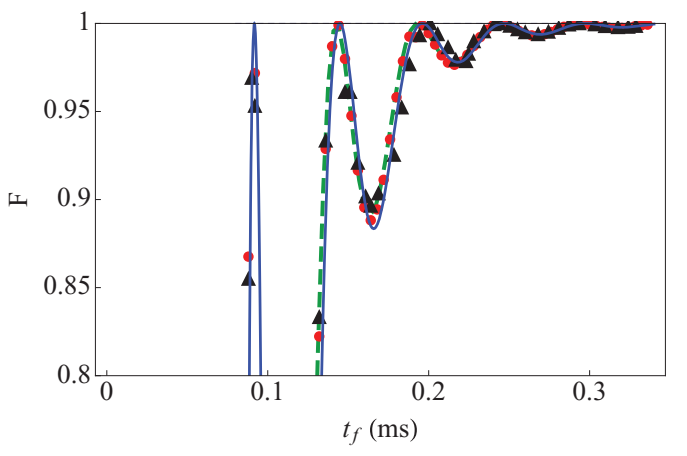

FIG. 1. (Color online) Fidelity of the anharmonic system vs final time $t_{f}$ following the inverse engineering trajectory using second-order perturbation theory (blue thick line), 1D dynamics for the initial ground state of the harmonic oscillator (red dotted line), 1D dynamics for the initial ground state of the perturbed 1D Hamiltonian (green dashed line), 2D dynamics for the initial ground state of the 2D Hamiltonian (filled triangles). $M=2 m=29.93 \times 10^{-27} \mathrm{~kg}$ corresponding to ${ }^{9} \mathrm{Be}^{+}$ions, $\omega /(2 \pi)=20 \mathrm{kHz}, d=370 \mu \mathrm{m}$, $r_{e}=62 \mu \mathrm{m}$, and $\beta=10^{6} \mathrm{~m}^{-2}$.

We shall now study the effect of each perturbation separately. The quadratic perturbation amounts to having designed the trap trajectory with the "wrong" trap frequency and, as we will see, is the dominant perturbation except for very short times. The influence of the quartic perturbation was analyzed in [10] but only with a much less accurate first-order approach. The effect of the two perturbations is quite different as seen in Fig. 2. The quadratic perturbation provides a fidelity almost identical to that of the total perturbation, reproducing its oscillations and peak times. The quartic perturbation alone leads to a sudden growth in the fidelity around a critical time $t_{f}^{c r}$, followed by fidelity 1 for longer final times. To estimate the behavior of $t_{f}^{c r}$ with respect to transport and potential parameters we note that the maximum of $\left|Q_{c}(t)-Q_{0}(t)\right|$ is $10 d /\left(\omega^{2} t_{f}^{2} 3^{1 / 2}\right)$. Comparing the quadratic

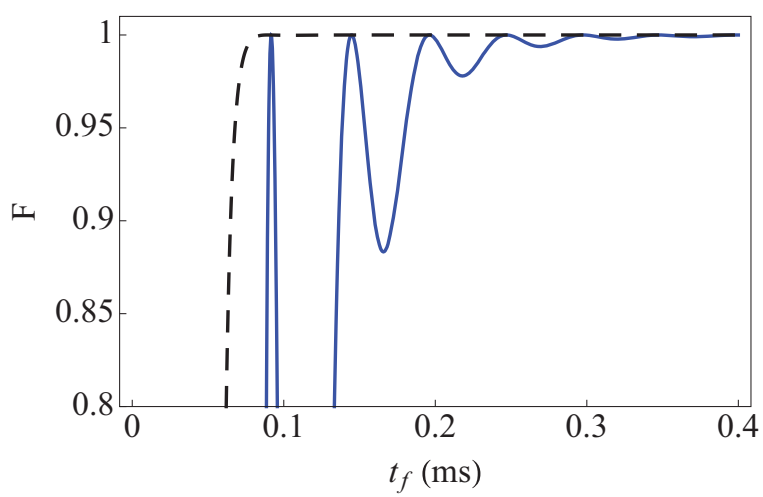

FIG. 2. (Color online) Fidelity vs final time $\left(t_{f}\right)$ for the secondorder perturbation theory, indistinguishable from an exact 1D quantum dynamical calculation (the initial state is the ground state of the perturbed harmonic oscillator) for the quadratic perturbation (blue thick line) in Eq. (14), and the quartic perturbation (black dashed line) in Eq. (14). Same parameters as in Fig. 1. and quartic contributions to the potential there, we get

$$
t_{f}^{c r}=\alpha \frac{\beta^{1 / 4} d^{1 / 2}}{\omega},
$$

where $\alpha \approx 16.5$ is adjusted numerically. For the parameters of Fig. 2 this occurs for shorter times than the one corresponding to the first peak of the quadratic perturbation so the effect of the quartic perturbation is negligible.

Let us now analyze in more detail the quadratic perturbation alone. It implies one and two vibrational quanta as mentioned before. If we consider only $n \rightarrow n \pm 1$, the results are already very similar to the fidelity in Fig. 2. Since one-level transitions are dominant we can write an explicit approximate form for the fidelity based on them:

$$
\begin{aligned}
f_{n \pm 1, n}^{(1)}= & \frac{ \pm 360 i d \beta r_{e}^{2} e^{\mp \frac{1}{2} i t_{f} \omega} \sqrt{2(1+n) M \hbar}}{t_{f}^{5} \omega^{9 / 2}} \\
& \times\left[6 t_{f} \omega \cos \left(\frac{t_{f} \omega}{2}\right)+\left(t_{f}^{2} \omega^{2}-12\right) \sin \left(\frac{t_{f} \omega}{2}\right)\right],
\end{aligned}
$$

(note the square root scaling with the mass). This amplitude is zero, and the fidelity one, when

$$
6 t_{f} \omega \cos \left(\frac{t_{f} \omega}{2}\right)+\left(t_{f}^{2} \omega^{2}-12\right) \sin \left(\frac{t_{f} \omega}{2}\right)=0 .
$$

There is a $\beta$-independent solution for, approximately, every oscillation period. This result also follows from a simple classical argument: Consider a classical trajectory $\widetilde{Q}_{c}(t)$ satisfying

$$
\frac{\ddot{\widetilde{Q}}_{c}}{\widetilde{\omega}^{2}}+\widetilde{Q}_{c}-Q_{0}(\omega)=0,
$$

where $Q_{0}(\omega)=Q_{0}(t ; \omega)$ is the trap trajectory calculated as before with $\omega$ [Eq. (4)] and $\tilde{\omega}=\omega \sqrt{1+6 \beta r_{e}^{2}}$ is an effective trap frequency, shifted with respect to $\omega$ because of the relative-CM coupling [see Eq. (13)]. Its energy for $\widetilde{Q}_{c}(0)=\dot{\widetilde{Q}}_{c}(0)=0$ is given by

$$
E_{e x}(t)=\frac{1}{2} M \dot{\tilde{Q}}_{c}^{2}(t)+\frac{1}{2} M \widetilde{\omega}^{2}\left[\widetilde{Q}_{c}(t)-Q_{0}(t)\right]^{2} .
$$

At time $t_{f}$ we have

$$
\begin{aligned}
E_{e x}\left(t_{f}\right)= & \frac{7200 d^{2} M\left(\omega^{2}-\tilde{\omega}^{2}\right)^{2}}{t_{f}^{10} \omega^{4} \tilde{\omega}^{8}} \\
& \times\left[6 t_{f} \tilde{\omega} \cos \left(\frac{t_{f} \tilde{\omega}}{2}\right)+\left(t_{f}^{2} \tilde{\omega}^{2}-12\right) \sin \left(\frac{t_{f} \tilde{\omega}}{2}\right)\right]^{2}
\end{aligned}
$$

The condition for a zero is the same as Eq. (20) substituting $\omega \rightarrow \tilde{\omega}$. This leads to a very small displacement (and dependence on $\beta$ ) of the zeros for our parameters. In Fig. 3 we represent $E_{0} /\left[E_{0}+E_{e x}\left(t_{f}\right)\right]$, which is indistinguishable from the curve where the excitation energy is calculated with quantum dynamics. We may conclude unambiguously that the oscillations are not quantum in nature.

Rather than adjusting the transport time to the discrete set of zeros, a better, more robust strategy that allows for a continuous set of final times is to design the trap trajectory taking into account the frequency shift. Changing $\omega \rightarrow \tilde{\omega}$ in Eq. (4) we get an adjusted trajectory $Q_{0}(t ; \tilde{\omega})$ for which 


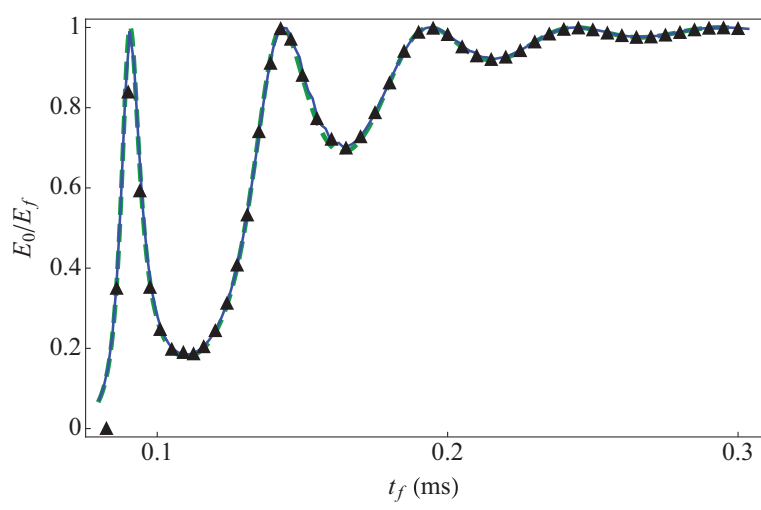

FIG. 3. (Color online) We plot $E_{0} /\left(E_{0}+E_{e x}\right)$, where $E_{0}$ is the ground-state energy for the 1D Hamiltonian and $E_{e x}$ is the excitation energy after the transport, for the 1D quantum evolution (blue solid line), 2D quantum evolution (black triangles), and a single classical trajectory (green dashed line). Same parameters as in Fig. 1.

$E_{e x}\left(t_{f}\right)=0$ by construction for any $t_{f}$. Similarly, $Q_{0}(t ; \tilde{\omega})$ gives fidelity one for all $t_{f}$ in the 1D model, if only the quadratic perturbation is considered. In the protocol based on $Q_{0}(t ; \tilde{\omega})$, the only disturbance comes from the quartic term that sets the speed limitation given by Eq. (18). Figure 4 shows the impressive results of this simple approach. In practice, $\tilde{\omega} /(2 \pi)$ may be measured as the effective CM-mode frequency.

Higher, more realistic trap frequencies lead to similar results but for a larger $\beta$. Simple estimates of the fidelity or excitation may be drawn from Eqs. (19) or (22). Figure 5 depicts the classical excitation energy of Eq. (22) for a realistic trap frequency and different values of $\beta$ using the (unshifted) $\omega$ in $Q_{0}(t)$. Notice that for these large- $\beta$ values the times of minimum excitation do change with $\beta$, and that, for the adjusted trajectory $Q_{0}(t ; \tilde{\omega}), E_{e x}\left(t_{f}\right)=0$ as before.

\section{FULL 2D ANALYSIS}

We have also examined the evolution of the state according to the full two-dimensional Hamiltonian (7), without freezing the relative motion, using a $2 \mathrm{D}$ split-operator method to simulate quantum dynamics. The computation is performed in the trap frame to reduce the numerical grid size. Figure 1 shows that the quantum fidelities of the 1D model are in very

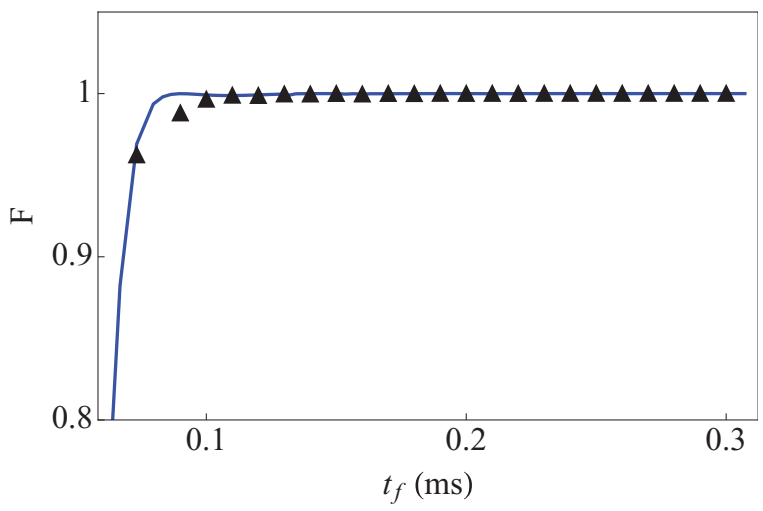

FIG. 4. (Color online) Fidelity vs final time $t_{f}$ for adjusted trap trajectories $Q_{0}(t ; \tilde{\omega})$. The initial condition is the ground state. 1D: blue solid line; 2D: filled triangles. Same parameters as in Fig. 1.

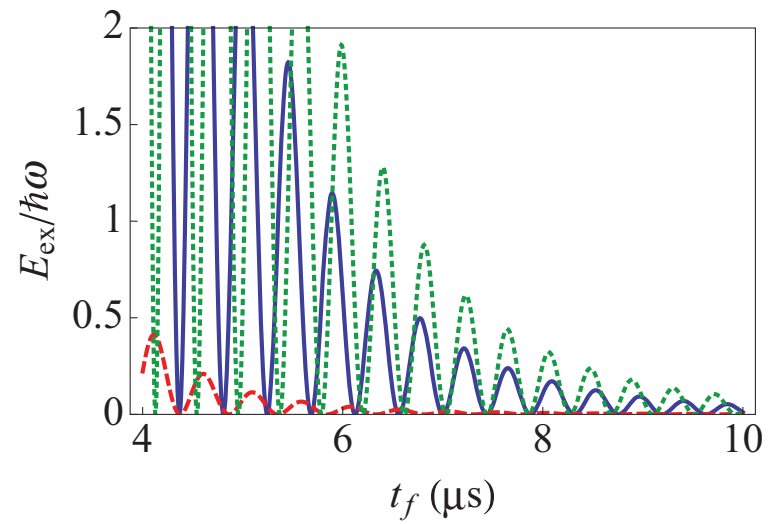

FIG. 5. (Color online) Motional excitation vs final time. $M=2 m=29.93 \times 10^{-27} \mathrm{~kg}, \omega /(2 \pi)=2 \mathrm{MHz}, d=370 \mu \mathrm{m}$ for the three cases and $\beta=6.4 \times 10^{9} \mathrm{~m}^{-2}, r_{e}=2.807 \mu \mathrm{m}$ (solid blue line), $\beta=10^{9} \mathrm{~m}^{-2}, r_{e}=2.883 \mu \mathrm{m}$ (red dashed line), and $\beta=10^{10} \mathrm{~m}^{-2}, r_{e}=2.764 \mu \mathrm{m}$ (green dotted line). The middle value of $\beta\left(6.4 \times 10^{9}\right)$ is chosen so that at $t_{f}=8 \mu$ s the excitation is similar to the one seen experimentally in [12]. The trap trajectory is given by Eq. (4). If instead the adjusted trajectory $Q_{0}(t ; \tilde{\omega})$ is used, then $E_{e x}\left(t_{f}\right)=0$.

good agreement with the fidelities calculated for 2D dynamics. Figure 3 shows energy ratios for $1 \mathrm{D}$ and $2 \mathrm{D}$ calculations. To compare them on equal footing in $2 \mathrm{D}$, the minima of the potential and the ground-state relative energy are subtracted. Again, the $1 \mathrm{D}$ and $2 \mathrm{D}$ quantum calculations are remarkably close to each other. 2D calculations may also be found in Fig. 4 for the transport designed using a shifted frequency. They confirm the excellent performance of this strategy with respect to the anharmonic perturbation.

\section{DISCUSSION}

For two ions in a harmonic trap, the relative motion is uncoupled to the CM motion. They may be transported faster than adiabatically treating the center of mass (CM) as a single particle and applying different shortcuts to adiabaticity. For anharmonic traps, CM and relative motion are coupled. A 1D model for the CM has been first worked out based on a single relative-motion mode or, equivalently, freezing the relative coordinate. The full 2D quantum calculations show excellent agreement with this model in the parameter range studied. It is possible to achieve fast and faithful transport for an arbitrary trap shape by compensating for the inertial force in the trap frame with a linear potential. That may be difficult in practice so other strategies to get high fidelities have been explored. For a quartic anharmonicity, the effective 1D potential includes a quartic and a quadratic perturbation. The latter is usually dominant except for very short transport times. If the trap trajectory is the one designed for the unperturbed (harmonic) trap, the quartic perturbation alone implies a sharp increase to one of the fidelity, while the quadratic perturbation induces (classical) fidelity oscillations with respect to the final time $t_{f}$. Taking into account the shift in the effective trap frequency due to the coupling, the trap trajectory is much more robust and the effect of the quadratic perturbation is canceled.

Other aspects worth investigating for future work are the effects of different types of noise [16] and other anharmonic forms such as cubic or time-dependent perturbations $[5,17]$. 
Variations of the trap frequency $\omega$ and anharmonicity factor $\beta$ with time could be affected by random and/or systematic perturbations. We expect that techniques similar to those applied in $[16,24]$ will be instrumental in designing robust trajectories. We also intend to study other relevant systems such as pairs of different ions as well as transport of four or more ions [25]. For more than three ions, quantum dynamical simulations are quite challenging but classical methods should provide a good guidance, as shown here for two ions.

\section{ACKNOWLEDGMENTS}

We are grateful to A. Ruschhaupt, D. Leibfried, and U. Poschinger for useful comments. We acknowledge funding by Grants No. IT472-10 and No. FIS2009-12773-C02-01, and the UPV/EHU Program UFI 11/55. M.P. acknowledges a fellowship by UPV/EHU.

\section{APPENDIX: $N$-ION TRANSPORT}

In this Appendix, we show that for $N$ equal ions in a harmonic trap the trap trajectory appears only in the CM part. In a harmonic trap with $N$ equal ions, the Hamiltonian is given by $N$ coordinates for the positions of each of the ions $\left(q_{1}, q_{2}, q_{3}, \ldots, q_{N}\right)$, and the corresponding momenta

$$
\begin{aligned}
\widehat{H}\left(\left\{\widehat{\mathrm{q}}_{i}, \widehat{\mathrm{p}}_{i}\right\}\right)= & \frac{1}{2 m} \sum_{i=1}^{N} \widehat{\mathrm{p}}_{i}^{2}+\frac{1}{2} m \omega^{2} \sum_{i=1}^{N}\left(\widehat{\mathrm{q}}_{i}-Q_{0}\right)^{2} \\
& +\sum_{i=1}^{N-1} \sum_{j=i+1}^{N} \frac{C_{c}}{\widehat{\mathrm{q}}_{i}-\widehat{\mathrm{q}}_{j}} .
\end{aligned}
$$

In coordinate space, $\mathrm{q}_{1}>\mathrm{q}_{2}>\cdots>\mathrm{q}_{N-1}>\mathrm{q}_{N}$ because of the strong Coulomb repulsion. We now define a CM and relative coordinates and momenta

$$
\begin{aligned}
& \widehat{Q}=\frac{1}{N} \sum_{i=1}^{N} \widehat{\mathrm{q}}_{i}, \quad \widehat{P}=\sum_{i=1}^{N} \widehat{\mathrm{p}}_{i}, \\
& \widehat{r}_{i}=\frac{\widehat{\mathrm{q}}_{i}-\widehat{\mathrm{q}}_{i+1}}{N}, \quad \widehat{p}_{i}=\widehat{\mathrm{p}}_{i}-\widehat{\mathrm{p}}_{i+1}, \quad i=1,2, \ldots, N-1,
\end{aligned}
$$

corresponding to the inverse transformation

$$
\begin{aligned}
& \widehat{\mathrm{q}}_{i}=\widehat{Q}+\sum_{j=1}^{N-i} j \widehat{r}_{N-j}-\sum_{k=1}^{i-1} k \widehat{r}_{k}, \\
& \widehat{\mathrm{p}}_{i}=\widehat{P}+\frac{1}{N} \sum_{j=1}^{N-i} j \widehat{p}_{N-j}-\frac{1}{N} \sum_{k=1}^{i-1} k \widehat{p}_{k} .
\end{aligned}
$$

The Hamiltonian in the new coordinates is

$$
\begin{aligned}
\widehat{H}= & \frac{\widehat{P}^{2}}{2 M}+\frac{1}{2} M \omega^{2}\left(\widehat{Q}-Q_{0}\right)^{2} \\
& +\frac{1}{2 N M} \sum_{i=1}^{N}\left[\left(\sum_{j=1}^{N-i} j \widehat{p}_{N-j}\right)^{2}+\left(\sum_{k=1}^{i-1} k \widehat{p}_{k}\right)^{2}\right. \\
& \left.-2 \sum_{j=1}^{N-i} \sum_{k=1}^{i-1} j k \widehat{p}_{N-j} \widehat{p}_{k}\right] \\
& +\frac{1}{2 N} M \omega^{2} \sum_{i=1}^{N}\left[\left(\sum_{j=1}^{N-i} \widehat{r}_{N-j}\right)^{2}+\left(\sum_{k=1}^{i-1} k \widehat{r}_{k}\right)^{2}\right. \\
& \left.-2 \sum_{j=1}^{N-i} \sum_{k=1}^{i-1} j k \widehat{r}_{N-j} \widehat{r}_{k}\right] \\
& +\frac{C_{c}}{N}\left(\sum_{i=1}^{N-1} \frac{1}{\widehat{r}_{i}}+\sum_{i=1}^{N-2} \sum_{j=i+1}^{N-1} \frac{1}{\sum_{k=i}^{j} \widehat{r}_{k}}\right)^{\prime}
\end{aligned}
$$

where $M=N m$. As for two ions, the Hamiltonian can be written as the sum of two terms $\widehat{H}=\widehat{H}_{\mathrm{cm}}(\widehat{Q}, \widehat{P})+\widehat{H}_{r}\left(\left\{\widehat{r}_{i}, \widehat{p}_{i}\right\}\right)$, where $\widehat{H}_{\mathrm{cm}}$ has the same form as that of a single particle driven in a harmonic trap, and $\widehat{H}_{r}$ depends only on $N-1$ relative coordinates and their corresponding momenta. It does not depend on the trap trajectory $Q_{0}(t)$, so this system can be transported without final excitations by following any shortcut-to-adiabaticity trap trajectory for a particle of mass $M$.
[1] D. Kielpinski, C. Monroe, and D. J. Wineland, Nature (London) 417, 709 (2002).

[2] M. Rowe et al., Quantum Inf. Comput. 4, 257 (2002).

[3] R. Reichle, D. Leibfried, R. B. Blakestad, J. Britton, J. D. Jost, E. Knill, C. Langer, R. Ozeri, S. Seidelin, and D. J. Wineland, Fortschr. Phys. 54, 666 (2006).

[4] H.-K. Lau and D. F. V. James, Phys. Rev. A 85, 062329 (2012).

[5] S. Schulz, U. Poschinger, K. Singer, and F. Schmidt-Kaler, Fortschr. Phys. 54, 648 (2006).

[6] E. Torrontegui, X. Chen, M. Modugno, S. Schmidt, A. Ruschhaupt, and J. G. Muga, New J. Phys. 14, 013031 (2012).

[7] A. Couvert, T. Kawalec, G. Reinaudi, and D. Guéry-Odelin, Europhys. Lett. 83, 13001 (2008).
[8] M. Murphy, L. Jiang, N. Khaneja, and T. Calarco, Phys. Rev. A 79, 020301(R) (2009).

[9] S. Masuda and K. Nakamura, Proc. R. Soc. A 466, 1135 (2010)

[10] E. Torrontegui, S. Ibáñez, X. Chen, A. Ruschhaupt, D. GuéryOdelin, and J. G. Muga, Phys. Rev. A 83, 013415 (2011).

[11] X. Chen, E. Torrontegui, D. Stefanatos, Jr-Shin Li, and J. G. Muga, Phys. Rev. A 84, 043415 (2011).

[12] R. Bowler, J. Gaebler, Y. Lin, T. R. Tan, D. Hanneke, J. D. Jost, J. P. Home, D. Leibfried, and D. J. Wineland, Phys. Rev. Lett. 109, 080502 (2012).

[13] A. Walther, F. Ziesel, T. Ruster, S. T. Dawkins, K. Ott, M. Hettrich, K. Singer, F. Schmidt-Kaler, and U. Poschinger, Phys. Rev. Lett. 109, 080501 (2012). 
[14] C. Roos, Physics 5, 94 (2012).

[15] E. Torrontegui, S. Ibáñez, S. Martínez-Garaot, M. Modugno, A. del Campo, D. Guéry-Odelin, A. Ruschhaupt, X. Chen, and J. G. Muga, Adv. At., Mol., Opt. Phys. 62, 117 (2013).

[16] A. Ruschhaupt, X. Chen, D. Alonso, and J. G. Muga, New J. Phys. 14, 093040 (2012).

[17] J. P. Home, D. Hanneke, J. D. Jost, D. Leibfried, and D. J. Wineland, New J. Phys. 13, 073026 (2011).

[18] N. W. Evans, J. Math. Phys. 31, 600 (1990).

[19] M. Karlovini, G. Pucacco, K. Rosquist, and L. Samuelsson, J. Math. Phys. 43, 4041 (2002).
[20] S. Masuda, Phys. Rev. A 86, 063624 (2012).

[21] E. Torrontegui, S. Martínez-Garaot, A. Ruschhaupt, and J. G. Muga, Phys. Rev. A 86, 013601 (2012).

[22] W. H. Klink, Ann. Phys. (NY) 260, 27 (1997).

[23] E. Torrontegui, X. Chen, M. Modugno, A. Ruschhaupt, D. Guéry-Odelin, and J. G. Muga, Phys. Rev. A 85, 033605 (2012).

[24] X.-J. Lu, X. Chen, A. Ruschhaupt, D. Alonso, S. Guérin, and J. G. Muga, Phys. Rev. A 88, 033406 (2013).

[25] J. P. Home, D. Hanneke, J. D. Jost, J. M. Amini, D. Leibfried, and D. J. Wineland, Science 325, 1227 (2009). 